\title{
A replication and extension of the Eriksen and Steffy (1964) experiment on short-term visual storage'
}

RALPH NORMAN HABER ${ }^{2}$

UNIVERSITY OF ROCHESTER

\begin{abstract}
Eriksen and Steffy (1964) were critical of short-term visual storage effects (STVS) because they were unable to find any evidence for them. In their first experiment they found instead great interference in performance over the same ISIs in which STVS is usually found. Their second experiment eliminated the interference by avoiding a bright second flash, but it still produced no evidence for STVS. Keele and Chase (1967) demonstrated that Eriksen and Steffy's second condition failed to find STVS because the memory load was too small, and perhaps because the luminance was too low. However, the design of Eriksen and Steffy's second experiment is not the one typically used to find STVS. Eriksen and Steffy's first experiment was replicated here, and a second condition was added in which each $S$ was also tested in a light adapted version. Interference was found in the former, as Eriksen and Steffy also found, but not in the latter. Little STVS was found in either of these conditions, presumably for reasons similar to those demonstrated by Keele and Chase.
\end{abstract}

Eriksen and Steffy (1964) presented Ss briefly with a display of six Xs and Os arranged around an imaginary clock face. This was followed by an indicator, which pointed to a spot where one of the six items had been. The $S$ was required to report that item. In their first experiment, the Ss were dark adapted, except for a dim fixation point. The target and indicator fields were each $3.7 \mathrm{ft}-\mathrm{L}$. Here they found that as the interstimulus intervals (ISI) between the target and indicator increased from 0 to $400 \mathrm{msec}$, accuracy of identification also increased-a result opposite in direction to those of Sperling (1960) and Averbach and Coriell (1961). Eriksen and Steffy's interpretation was that the luminance of the indicator was summating with that of the target field thereby reducing the contrast of the target item and, presumably also, the indicator.

In their second and third experiments, they used a luminous indicator against a dark background so that no luminance summation could occur between the indicator and the target. Here they found essentially no change in accuracy over $700 \mathrm{msec}$, although in the third experiment, with more extensive data on each $S$, rather large individual differences were found.

Eriksen and Steffy concluded from these experiments that no evidence for a short-term visual storage effect (STVS) was found, since in no condition was accuracy, based on a partial report, greater for short as compared to longer ISIs. Further, they implied that interference effects such as those found in their Experiment 1 should prevent the demonstration of an STVS. They seemed to feel that perhaps the discrepancy with the literature could be explained by the lower luminance levels they used as compared to those of Sperling, and Averbach and Coriell.

The Eriksen and Steffy experiments are inappropriate for the demonstration of STVS effects for two independent reasons: (1) the memory load contained in their display is so small that no loss in accuracy should be expected even with a long delay in the indicator; and (2) luminance summation creates interference at those ISIs in which STVS normally is found, at least for Experiment 1. The first point has been documented nicely by Keele and Chase (1967). They replicated Experiment 2 but with larger memory loads-all letters rather than just $X$ and $O$, and 10 positions rather than just six. Under these conditions they found accuracy decreased in the ISI range from 0 to $250 \mathrm{msec}$. They also varied the luminance of the target $(3.7,16$, and $70 \mathrm{ft}-\mathrm{L})$ and found more STVS at the higher luminance summation should have occurred, they concluded that the Eriksen and Steffy design was deficient because of the small demands on memory.

This still leaves the luminance summation effect in Experiment 1 to be reconciled with the STVS literature. Why did they fail to find any STVS when several other investigators had already demonstrated STVS in a two-flash design? The experiment reported here is based on the assumption that the interference Eriksen and Steffy found had nothing to do with the absolute luminance levels, as they had speculated, but rather with the presence or absence of the background illumination. When the $S$ is dark-adapted and then presented with two successive flashes (as in their Experiment 1), the luminance of the two can summate, thus reducing the contrast of the target and of the indicator as well. However, if the $\mathbf{S}$ is light-adapted to the same level as the flashes, then while each flash can summate with the background, thereby reducing the contrast of each slightly, the two flashes cannot summate together. Thus, the presence of the second flash cannot create any interference with the first. Therefore, under such conditions, if an STVS effect is occurring, no interference will mask it. When the second stimulus is luminous, so that no background luminance is present, no luminance-summation contrast reduction is possible since there is no background luminance to summate with the first stimulus. It is for this reason that their Experiment 2 is free of interference effects, and it should not matter whether $S$ is light- or dark-adapted. (Keele and Chase showed an STVS effect with dark-adapted Ss.) However, in Experiment 1, in which the second stimulus is on a bright background, the critical variable should be whether the background illumination during the ISI is bright or dark. To test this, Experiment 1 was replicated and compared to a new condition in which the background and ISI was also at $3.7 \mathrm{ft}-\mathrm{L}$, the same as the target and indicator fields.

\section{METHOD}

Two conditions were run, one of which (Dark) was identical to that of Eriksen and Steffy's Experiment 1, using even the same type of apparatus. The second condition (Light) was the same, except that the background was set also at $3.7 \mathrm{ft}-\mathrm{L}$, and was on at all times except during the display of the target and the indicator. Three Ss served in 20 1-h sessions, four for practice and 16 experimental sessions (eight Dark and eight Light), the latter alternating from session to session. Nine ISIs between the target and the indicator were used: simultaneous. $0,10,50,100,300$. 800,1600 , and $3200 \mathrm{msec}$. The $300 \mathrm{msec}$ ISI was used only in the first eight experimental sessions and the 3200 only in the last eight. The simultaneous ISI had the indicator drawn on the target stimulus so that only one channel was needed to display it. For the first eight experimental sessions the indicator channel, with a blank card in it, was also flashed at the same time, which increased the luminance of the field and thereby decreased the contrast of the indicator. In the second eight sessions no blank channel was used.

A threshold duration was found for each $S$ following the procedures of Eriksen and Steffy, except that it was set unintentionally at about $80 \%$ correct instead of $90-100 \%$ as they had used. The average duration for the three Ss was 15,20, and 16 msec, respectively. The same duration for each $S$ was used in the Light and Dark conditions, and for the target and the indicator. Each session contained 96 trials -2 targets by 6 positions by 8 ISIs. The $S$ was required to report the item indicated and its position, and to guess if neccessary. He was scored correct only if he named the correct item in the correct location.

\section{RESULTS AND DISCUSSION}

Figure 1 presents the results in per cent correct for each of the three Ss for the Light and Dark conditions, respectively, using the single channel simultaneous trials. An analysis of variance showed 

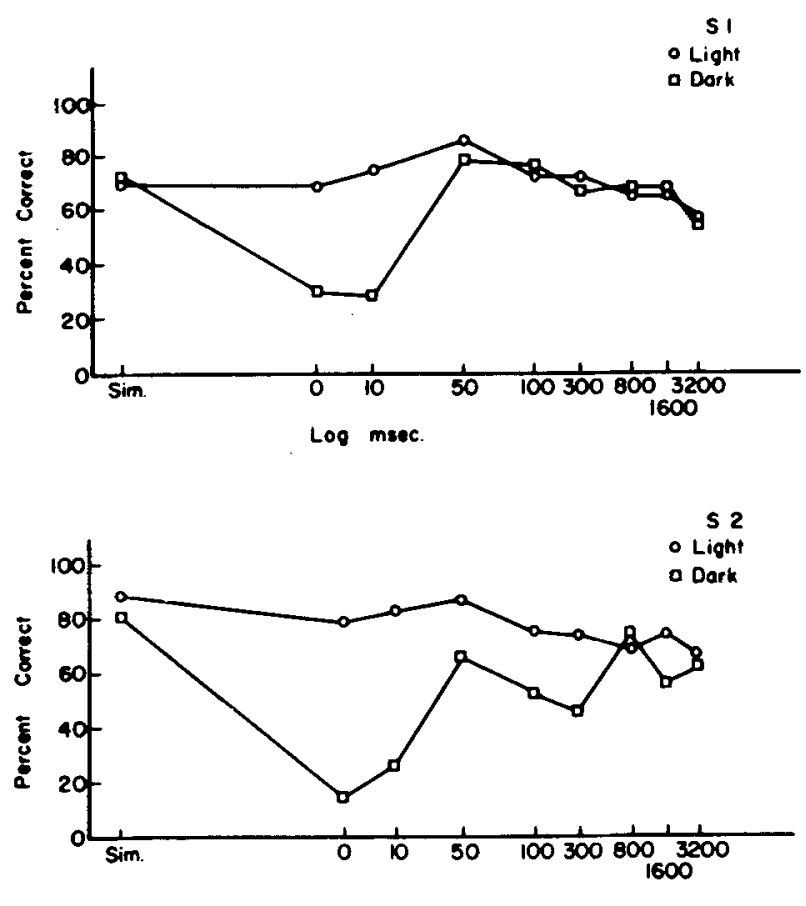

Log msec.

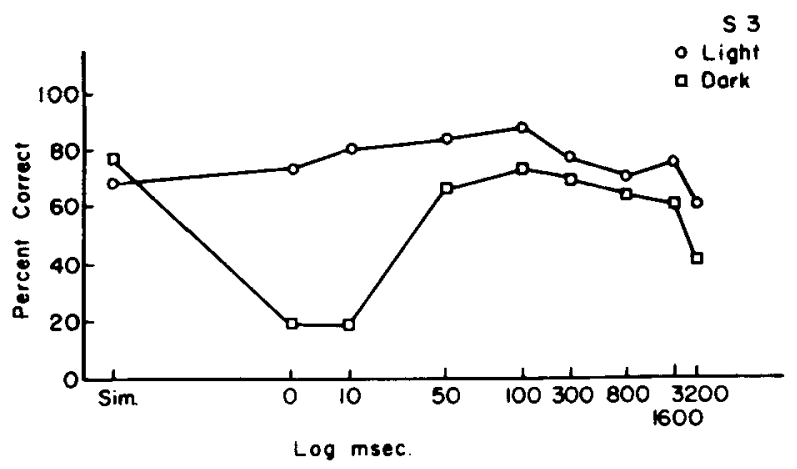

Fin. 1. Percentage of targets correctly reported by three Ss for the Light and Dark conditions, respectively. A log scale of time in miltiseconds is used, defined as onset-to-onset. The stimulus disploy is given an arbitrary asynchrony of $1 \mathrm{msec}$ for purposes of plotting $\log$ values.

significant main effects for ISI, for condition, and also for their interaction (all $\mathrm{p}<.001$ ). No main or interactive effects of Ss were significant. It seems apparent that most of the significant effects are accounted for by the vastly poorer accuracy in the dark conditions for the 0 and $10 \mathrm{msec}$ ISIs. Beyond an ISI of $50 \mathrm{msec}$ there is a significant linear decrease in performance $(p<.01)$ but it is not large, averaging only $15 \%$ drop over Ss and conditions.

These results support the hypothesis offered. Under the Dark condition interference is found for short ISIs, as shown by the sharp drop in accuracy. For the Light condition no interference is found. In both conditions, the STVS effect is very small, but Keele and Chase have already explained why it should be absent with this type of target.

The interference effect shown here for the Dark condition is far greater than that found by Eriksen and Steffy (see Fig. 2 for their comparable curve from Experiment 1). They found only a $30 \%$ drop, while an average interference of $60 \%$ was found here.

Two sources of interference are possible difficulty in seeing the target and difficulty in seeing the indicator. Eriksen (1966) has shown that luminance-summation effects are symmetrical for this type of task. Therefore, in the dark conditions, as the ISI decreases, the indicator should interfere with the perception of the target, and the target should interfere with the perception of the indicator. To compute the value of a chance level of performance, the probability of perceiving the target and the indicator must be taken into account: if the indicator is seen, the chance level of a correct guess is 0.50; if neither the indicator nor the target are seen, chance will be $\mathbf{0 . 8 3}$. Because Eriksen and Steffy set baseline performance at $97 \%$, luminance-summation apparently did not reduce the perceptibility of either target or indicator as much as in this replication, in which baseline performance was $78 \%$. They do not mention the accuracy with which the indicator alone was reported; but it can be assumed that it was nearly perfect at all ISIs. In the replication for the dark condition, accuracy of reporting just the indicator ranged from $100 \%$ (for the simultaneous presentation, and ISIs of $50 \mathrm{msec}$ or greater) to a mean of $30 \%$ for the 0 and $10 \mathrm{msec}$ ISIs. Thus, indicator accuracy parallels overall accuracy. If Fig. 2 is corrected for the far higher chance level (close to $50 \%$ ) in the Eriksen and Steffy experiment, as compared to the much lower value in the replication, for the 0 to $10 \mathrm{msec}$ ISIs, the discrepancy. between the Dark condition curves of Fig. 1 with Fig. 2 would be greatly reduced.

The simultaneous trials in Fig. 1 are from the second eight experimental sessions with the indicator channel off. This produced scores of $75 \%$ and $77 \%$ averaged over the three Ss for the Light and Dark conditions, respectively. The comparable scores (not drawn on Fig. 1) from the first eight experimental sessions in which the indicator channel was left on, thereby theoretically adding interference through contrast reduction, averaged $78 \%$ for the Light condition and $35 \%$ for the Dark condition. Presumably, for the Light condition in this single flash. case, if some luminance summation occurs between the background and the target, adding more light from a second stimulus channel on top of the target has little effect. The fact that the accuracy does not change from simultaneous ISI to 0 ISI shows that light added temporally adjacent to the target also has little effect. However, in the Dark condition, which normally has no luminance summation on the simultaneous trials, light added from another channel greatly reduces accuracy. But, there is no further drop from the simultaneous ISI trials (with extra light) to the 0 ISI trials. Thus, adding the light on top of the target or temporally next to the target seems fairly equivalent. This can account for the relatively large drop in accuracy that Eriksen and Steffy found between their simultaneous (with no extra light added) ISI and the 0 ISI condition.

For S1, the differences between Light and Dark conditions end at $50 \mathrm{msec}$, while S2 and S3 show a slightly lower accuracy on the Dark condition long after that. The trend seems apparent, however, that the luminance summation effects in this situation are not large after $50 \mathrm{msec}$, at least as compared to their magnitude at shorter intervals.

In summary, this experiment has shown that performance will decrease presumably due to luminance summation between two flashes when the $S$ is dark-adapted but not when light-adapted.

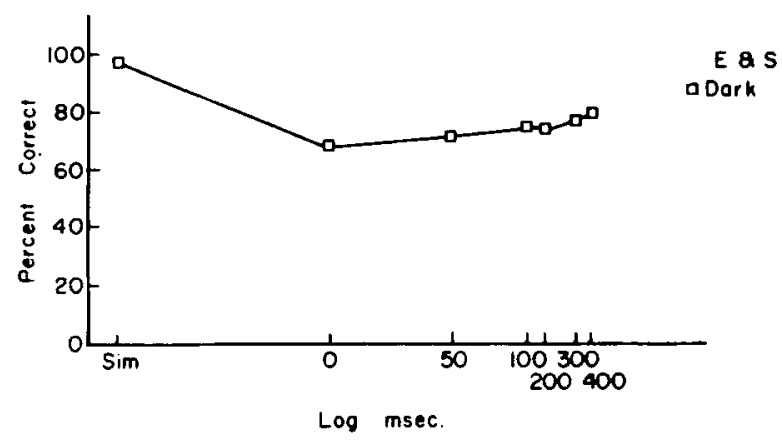

Fig. 2. Average percentage of targets correctly reported by the 10 best $\mathrm{Ss}$ in Eriksen and Steffy's Experiment 1. Data taken from Eriksen and Steffy. 
Thus, Eriksen and Steffy's criticisms of STVS effects is some what unfair, since STVS uses light-adapted Ss in which little interference would be expected. It seems apparent that if the indicator in an STVS experiment is drawn on a lighted background, and the ISI is dark, then interference must result. Such interference can be avoided either with the use of a light-adapted $\mathrm{S}$, or by removal of the lighted background of the indicator presentation. ${ }^{3}$ This result, plus that of Keele and Chase (1967) regarding memory loads and luminance level help to define the boundary conditions needed to demonstrate a STVS effect.

\section{REFERENCES}

AVERBACH, E., \& CORIELL, A. S. Short-term memory in vision. Bell System Technical Journal, 1961, 40, 309-328.

ERIKSEN, C. W. Temporal luminance summation effects in backward and forward masking. Perception \& Psychophysics, 1966, 1, 87-92.

ERIKSEN, C. W., \& STEFFY, R. A. Short-term memory and retroactive interference in visual perception. Joumal of Experimental Psychology, $1964,68,423-434$.
KEELE, S. W., \& CHASE, W. G. Slırt-term visual storage. Perception \& Psychophysics, 1967, 2, 383-386.

SPERLING, G. The information available in brief visual presentations. Psychological Monographs, 1960, 74, 1-29.

\section{NOTES}

1. This research was supported in part by grants from the National Science Foundation (GB 2909 and GB 5910) and the United States Public Health Service (MH 10753). I would like to thank Miss Barbara Herr for her assistance in running the $S s$ and participating in the analysis of the data.

2. Address: Department of Psychology, University of Rochester, Rochester, N. Y. 14627.

3. Of course, several different types of interference are possible in two flash experiments, especially metacontrast effects from closely adjacent or overlapping parts of the stimulus cord indicator. Such effects were not the concern of this paper.

(Accepted for publication August 9, 1968.) 